Los efectos del disfrute en la ejecución de instrumentos musicales Nora Blanca Leibovich, Mariel Gimenez y Florencia Martucci Epistemus - Revista de estudios en Música, Cognición y Cultura, 7(2), 27-56, e009, 2019 ISSN 1853-0494 | https://doi.org/10.24215/18530494e009 https://revistas.unlp.edu.ar/Epistemus

\title{
Los efectos del disfrute en la ejecución de instrumentos musicales
}

\section{Nora Blanca Leibovich, Mariel Gimenez y Florencia Martucci nleibovich@fibertel.com.ar}

\author{
Universidad de Buenos Aires (UBA) - Consejo Nacional de Investigaciones Científicas y Técnicas \\ (CONICET)
}

\section{Resumen}

En el presente estudio buscamos conocer los elementos que constituyen la experiencia de disfrute en relación a sus efectos en la actividad de ejecución musical. Conocer/explorar la experiencia de disfrute en el ejecutante. Realizamos un estudio exploratorio (cuali-cuantitativo) sobre la experiencia de disfrute en ejecutantes musicales. La pregunta formulada fue: ¿qué efecto crees que produce en tu vida disfrutar de la ejecución musical? A través del análisis de las frases de los participantes se identificaron diferentes experiencias psicológicas que fueron agrupadas en categorías específicas y sus componentes expresivos. Respondieron de manera escrita 207 ejecutantes musicales (81,15\% varones) de entre 16 y 55 años de Buenos Aires (Argentina). Los sujetos se categorizaron según edad, sexo, tipo de instrumento que ejecuta. El efecto del disfrute en la ejecución de un instrumento musical se encuentra significado como felicidad y relajación en el área emocional; mientras que tranquilidad, bienestar, satisfacción y despreocupación caracterizan el efecto del disfrute en el área cognitiva. A nivel motivacional se ha consignado que el efecto del disfrute en la ejecución musical es la realización personal principalmente. Estas cuestiones han sido rescatadas de los dichos de los y las participantes de la presente investigación, expresando estados complejos.

\section{Palabras Clave}

disfrute, experiencias positivas, música, instrumentistas. 


\title{
The effects of enjoyment on the performance of musical instruments
}

\begin{abstract}
In this study we seek to know the elements that constitute the experience of enjoyment in relation to its effects on the musical performance activity. Know/explore the experience of enjoyment in the performer. We will carry out an exploratory study (quali-quantitative) about the experience of enjoyment in musical performers. The asked question was: What effect do you think it has on your life to enjoy musical performance? Through the analysis of the participants' phrases, different psychological experiences were identified that were grouped into specific categories and their expressive components. 207 musical performers $(81.15 \%$ male) between 16 and 55 years old from Buenos Aires (Argentina) responded in writing. The 207 subjects were categorized according to age, sex, type of instrument they performed. The effect of enjoyment in the performance of a musical instrument is found as happiness and relaxation in the emotional area; while tranquility, wellbeing, satisfaction and carefreeness characterize the effect of enjoyment in the cognitive area. At the motivational level, it has been stated that the effect of enjoyment on musical performance is personal fulfillment mainly. These issues have been recovered from the speechs of the participants of the present investigation, expressing complex states.
\end{abstract}

\section{Key Words}

enjoyment, positive experiences, music, instrument performers. 


\section{Introducción}

La principal motivación para involucrarse con la música en el mundo occidental contemporáneo es facilitar las transacciones involucradas en la formulación, presentación y afirmación de las múltiples dimensiones de las identidades individuales y grupales. Se han explorado las dimensiones afectivas de los significados específicamente musicales.

El trabajo de DeNora (2000) sugiere que la música puede ser significativa para el oyente en la regulación de sus emociones y estados de ánimo en la vida cotidiana. Los hallazgos de DeNora se ven reforzados por los de Sloboda, O’Neill e Ivaldi (2001), quienes emplearon un enfoque innovador (el método de muestreo de experiencia) para la naturaleza y el significado de la experiencia de la música en la vida cotidiana. Encontraron que la música era particularmente significativa en contextos sociales e individuales. La eficacia de la música en la regulación de la emoción y el estado de ánimo era mayor en tales contextos.

$\mathrm{Al}$ examinar la literatura sobre el significado musical se observa que no hay consenso en cuanto a la mejor manera de abordar las cuestiones. La opinión mayoritaria de las disciplinas es que la música está imbuida de significados.

En esta heterogeneidad en relación a la cuestión del significado musical surgen, en la literatura teórica y empírica, representaciones bastante diferentes del significado musical. Se podría sugerir que este es un hecho inevitable debido a la difusividad del concepto que se está investigando.

La mayoría de los enfoques empíricos acerca del significado de la música toman como objeto de su investigación los procesos que intervienen en la escucha musical. Una notable excepción en este último dominio es el trabajo de Finnegan (1989), la investigación etnomusicológica indica que la música se concibe mejor como un modo de interacción que como objeto de la percepción auditiva. Las ciencias psicológicas necesitan encontrar maneras para abordar el estudio de los significados que son inherentes o emergentes en los procesos de interacción musical.

El desarrollo de la comprensión musical y actividades musicales revela un complejo patrón de hallazgos. Los debates en el campo giran en torno a las influencias de la naturaleza y la cultura, la edad y la experiencia.

Lamont (2011) ha defendido la utilidad del contexto en la comprensión de aspectos que influyen en los niños y sus niveles de compromiso con la música. 
Incluye los microcontextos, del hogar y la escuela, hasta los macrocontextos, de las creencias dominantes en la sociedad. Este tipo de enfoques proporciona medios más prácticos de identificar y explorar los contextos significativos en la vida de los niños y adolescentes en relación a la música. Esta forma nos permite conocer los motivos que ayudan al desarrollo musical. Una consideración más completa del contexto también puede ayudar a abordar los desafíos empíricos del compromiso y motivación para la música.

En el caso de la música, nos enfrentamos con la paradoja de que las personas disfrutan escuchar música que evoca emociones negativas, es decir que disfrutan escuchar música que les provoca tristeza, melancolía o cualquier otro afecto de esa naturaleza (Schubert, 1996).

Un importante trabajo de conceptualización en relación a los aspectos que nos permiten generar y expresar nuestros juicios estéticos respecto a la música es el de Juslin (2016), para quien el juicio estético es un proceso donde se evoca una emoción en el oyente. Esto se debe al valor estético que le otorga el sujeto a la música. En primer lugar, el oyente adopta una actitud estética, que significa que el criterio estético (por ejemplo: belleza, novedad) influye en la música. Las entradas perceptuales, cognitivas y emocionales acerca del evento musical son filtradas de acuerdo al peso relativo del criterio subjetivo del escucha. La evaluación ocurre de una manera continua. La expresión de estos juicios puede ocurrir como episodios en cierto momento del tiempo, por ejemplo, en un momento significativo como en un final de solo de jazz. El resultado es que la música es juzgada extraordinariamente buena o mala (umbral estético) (Figura 1). En resumen, para Juslin existe una preclasificación como arte a través de las entradas perceptuales, cognitivas y emocionales que son filtradas de acuerdo al criterio estético dando lugar a los juicios estéticos. Los juicios expresados pueden ser positivos o negativos. Si son positivos, da lugar a la emoción. Si son negativos también generan una emoción, conectada o desconectada al umbral estético.

A continuación presentamos el modelo del proceso propuesto por Juslin: 


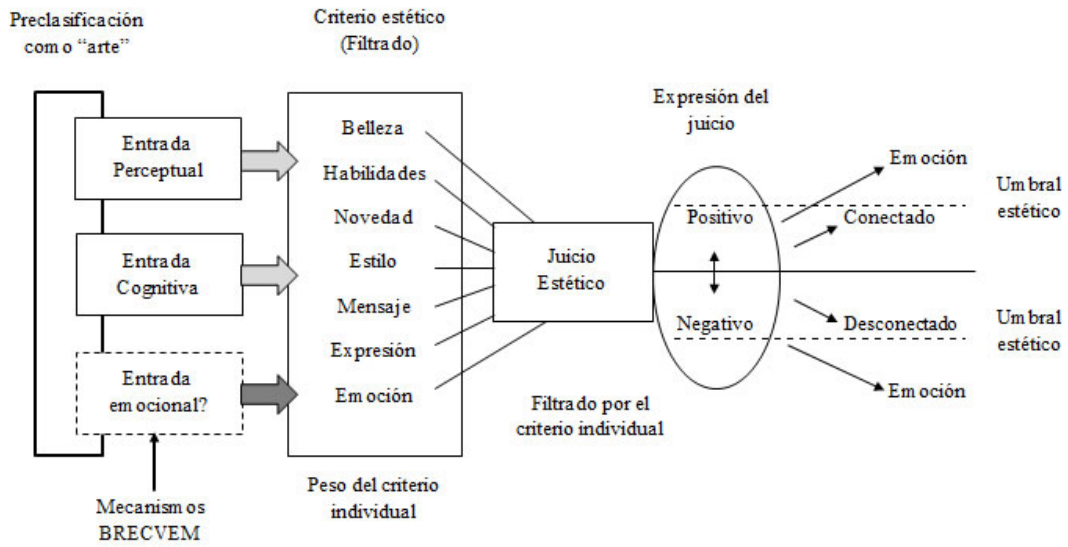

Figura 1. Modelo esquemático del proceso de juicio estético durante la escucha de la música. Adaptado de Juslin (2016).

Se asocia la música al disfrute tanto en la ejecución como en la escucha. Lo dicho hasta aquí está referido al sujeto en la escucha musical.

Debemos destacar que nuestro interés está centrado en observar la intensidad de la experiencia de disfrute en los propios actores y generadores de música: los ejecutantes de instrumentos musicales.

Desde el punto de vista psicológico y en el campo del estudio de las experiencias subjetivas positivas, se ha identificado el flow (fluir) (Csikszentmihalyi y Csikszentmihalyi, 1975) como una experiencia subjetiva óptima, siendo este un estado complejo y altamente estructurado de profundo involucramiento, absorción y disfrute. En esta teorización se asume al disfrute como un producto de la experiencia óptima de fluidez. Estudios posteriores postulan que el componente cognitivo de la experiencia es el más estable en diversas culturas, edades y actividades, siendo el componente afectivo el que varía (Delle Fave, Massimini y Bassi, 2011).

Se puede definir el disfrute como el estado que se produce cuando un sujeto encuentra una actividad automotivante y así se compromete por razones intrínsecas más que por recompensa extrínseca (Moon y Kim, 2001). El término disfrute (enjoyment) ha sido utilizado para indicar una disposición positiva general hacia el gusto por una actividad o contenido. Posee componentes afectivos, cognitivos y comportamentales que operan a un nivel evaluativo y experiencial de la persona (Nabi y Krcmar, 2004). 
Obras clásicas relacionadas con la antropología y la etnomusicología ubican a la música como un fenómeno universal. Aristóteles ubica a la música en una posición especial respecto a otras artes ya que no imita objetos externos, sino pasiones y virtudes (Kohut y Levarie, 1950). También se ha estudiado al disfrute como predictor y como producto de actividades físicas (Williams et al., 2006), probando sus asociaciones con la motivación y el sostenimiento de la actividad.

Con relación a los efectos de la música y al disfrute en particular, Schneider (1921) trataba de definir el disfrute musical asimilándolo a una sensación de placer desde una mirada muy simple.

Debemos destacar nuevamente que lo expuesto anteriormente está básicamente centrado en el oyente. Nuestra intención es centrarnos en los protagonistas del evento musical, los ejecutantes de instrumentos musicales. Creemos ahí está la novedad de este aporte.

En tanto vamos adentrándonos en el estudio de las personas que ejecutan instrumentos musicales, muchos interrogantes emergen. En el presente estudio, de corte exploratorio, comenzaremos incluyendo la indagación de variables sociodemográficas básicas como la edad, el sexo y, con relación a la práctica, tomaremos en cuenta el tipo de instrumento.

Respecto al sexo de los ejecutantes musicales, estudios previos han demostrado que la elección del instrumento está sesgada por estereotipos de género. Análisis lineales revelaron que los niños y las niñas tienen ideas similares sobre qué instrumentos no deberían tocar los miembros de cada sexo (O’Neill y Boultona, 1996). Asimismo, estudios más recientes han profundizado en el análisis de la categoría de género en la ejecución musical, respecto a los roles que se asumen en la escena musical. Se han encontrado prácticas discriminatorias en la socialización temprana diferenciada por sexo en la práctica musical, por ejemplo: dificultades de las mujeres en acceder y permanecer en un ambiente masculinizado y dificultades para las mujeres en articular la esfera privada con el desarrollo profesional (para una revisión ver Buscatto, 2016). A la luz de estos hallazgos, nos preguntamos si la experiencia de disfrute en la ejecución musical y los efectos que produce en los y las ejecutantes de instrumentos musicales son distintos.

En general en todas estas aproximaciones al estudio del disfrute en la ejecución musical, aun teniendo en cuenta variables como el sexo, la edad o el instrumento -como hemos mencionado anteriormente-, se refieren a los efectos del disfrute en términos de emociones positivas (contrastando con emociones negativas o ansiedad en la performance). Esta conceptualización aporta generalizaciones sobre el efecto del disfrute en la ejecución musical. 
¿Qué dimensiones psicológicas están presentes en el disfrute en la ejecución musical? ¿Qué esferas psicológicas se encuentran más comprometidas en esta experiencia? ¿Qué descriptores utilizan los y las ejecutantes para hablar de los efectos que pudiera tener el disfrute en la ejecución musical? ¿Existen diferencias por sexo, por edad o por instrumentos en los efectos que tiene la experiencia de disfrute en la ejecución musical?

Dado que el disfrute se considera un estado psicológico positivo relacionado con el flow procederemos a aclarar algunos aspectos.

\section{Reflexiones acerca del disfrute y su relación con el Flow}

La experiencia óptima de flow es un estado en el cual las personas se hallan tan involucradas en la actividad que están realizando que nada más parece importarles (Csikszentmihalyi y Csikszentmihalyi, 1988). Esta experiencia es tan agradable que las personas desean realizarla por el disfrute que les proporciona la actividad en sí misma, incluso cuando esta actividad pueda ser extremadamente difícil o peligrosa, o pueda no existir recompensa tangible (Csikszentmihalyi, 1993). Es así que el estudio del disfrute se relaciona con otras experiencias óptimas, como la de flow, y amplía las aproximaciones posibles al estudio de experiencias que complejizan nuestro involucramiento en actividades automotivadas que promueven el crecimiento personal.

Dada la riqueza que produce la experiencia de disfrute con relación a una actividad o comportamiento hemos elegido la ejecución de instrumentos musicales para su observación, incorporando al estudio de las experiencias subjetivas positivas el campo de la ejecución musical. La exploración de los aspectos psicológicos de los y las ejecutantes de instrumentos musicales es un campo novedoso en nuestro contexto sociocultural y académico.

El abordaje del presente trabajo es de corte cualitativo. Es materia prioritaria en la investigación psicológica la indagación de las concepciones desde los propios sujetos protagonistas y sus significados subjetivos dado que determinan en cierto grado su comportamiento. En este contexto, la mirada cualitativa brinda la oportunidad de indagar las percepciones de los grupos humanos sin restringirlas a categorías generadas en contextos muy distintos del propio investigador.

El concepto de disfrute debe ser investigado y analizado desde sus propios actores y contexto sociocultural. Los constructos psicológicos deben ser considerados construcciones histórico-sociales, imbuidas en una cultura que les imprime significados únicos. No es recomendable extrapolar a otros el uso de conceptos construidos y validados en otro medio social (Leibovich y Schmidt, 2010). Como 
hemos señalado anteriormente, tanto la conceptualización del disfrute como el conocimiento de las dimensiones que componen la experiencia y los efectos reportados por los y las ejecutantes, no han sido acabadamente abordados en nuestro contexto académico. Esta cuestión no sólo pone de manifiesto el aspecto novedoso de esta investigación, sino que pretende aportar a la comprensión de la ejecución musical. Un abordaje cualitativo implica la exploración de sentidos y significados desde las personas que protagonizan las experiencias que investigamos.

A partir de estas reflexiones este trabajo se propone explorar características del disfrute en ejecutantes de instrumentos musicales. Específicamente, se busca conocer/explorar qué efectos produce la experiencia de disfrute en el ejecutante.

\section{Método}

Se generó un instrumento psicológico denominado Inventario de la experiencia de disfrute en ejecutantes de instrumentos musicales (IDEM) para la evaluación de la experiencia de disfrute en la ejecución musical. A los participantes se les entregó el cuestionario en formato papel u online. Debían responder a la siguiente pregunta escrita de respuesta abierta: ¿qué efecto crees que produce en tu vida disfrutar de la ejecución musical?

\section{Participantes}

207 ejecutantes musicales (81,15\% varones) de entre 16 y 65 años de Buenos Aires (Argentina).

\section{Técnicas de producción de la información}

La tarea analítica constó de diferentes momentos. Para el análisis de las respuestas a la pregunta solicitada y las del cuestionario sociodemográfico se utilizó el programa ATLAS.ti, para el análisis de datos cualitativos.

Los 207 sujetos se categorizaron según sexo, rango de edad y tipo de instrumento que ejecuta. El 18,84\% eran mujeres y el $81,15 \%$ varones, distribuidos en los siguientes rangos de edades: 16 a 25 años (33,81\%), 26 a 35 años $(44,44 \%)$, 36 a 45 años (11,59\%), 46 a 55 años (7,24\%), 56 a 65 años $(2,89 \%)$. Con relación a la ejecución de instrumentos, el 69,56\% ejecutaba instrumentos de cuerdas, el $12,07 \%$ percusión, el 7,72\% piano, y el 10,62\% viento.

A través del análisis de las frases de los participantes se identificaron diferentes 
experiencias psicológicas. Posteriormente fueron agrupadas en categorías específicas y sus componentes expresivos.

Se realizó un análisis de contenido para la clasificación y definición de las categorías y se mantuvieron aquellas que resultaron claras de inferir y de aplicar, con relevancia conceptual, y resultantes del consenso entre los investigadores e investigadoras.

Todo el procedimiento fue orientado a establecer la credibilidad, dependencia y confirmabilidad de los datos (Hernández Sampieri, Fernández-Collado y Baptista, 2014), como criterios de rigor en la investigación cualitativa.

A continuación se presentan ejemplos representativos de respuestas obtenidas frente a la pregunta: ¿qué efecto crees que produce en tu vida disfrutar de la ejecución musical?

Se hace referencia en cada respuesta a la experiencia psicológica implícita, es decir, a los componentes expresivos. Posteriormente se definen las categorías que los vinculan.

\section{Cognitivos}

- Confianza: el disfrute en la ejecución fortalece la autoestima al producir un efecto de confianza en sí mismo. Ejemplo: "confianza en uno mismo" (Masculino, 18 años), "genera autoconfianza" (Femenino, 24 años).

- Concentración: al disfrutar de la ejecución se genera un estado mental de mayor concentración. Ejemplo: "me ayuda a concentrarme luego en otras cosas" (Femenino, 27 años), "produce agilizar la mente, ya que a medida que uno avanza va incorporando más conceptos musicales teóricos y prácticos, por lo tanto lo fuerza a uno a razonar, a usar la mente" (Masculino, 25 años).

- Despreocupación: el efecto de disfrutar genera un estado mental de liberación de preocupaciones y problemas durante el momento de la ejecución. Ejemplo: "es un cable a tierra de las cosas que a uno le pasan día a día" (Masculino, 24 años), "me libera de las preocupaciones por ese momento" (Femenino, 26 años).

- Tranquilidad: el disfrutar de la ejecución produce un estado de calma. Ejemplo: "estar más en paz conmigo mismo" (Masculino, 25 años), "me da tranquilidad, armonía" (Masculino, 45 años).

- Satisfacción: el efecto de disfrutar de la ejecución de un instrumento pro- 
duce un estado de satisfacción. Ejemplo: "me da mucha satisfacción, me ubica en mi lugar preferido" (Masculino, 40 años), "satisfacción plena" (Masculino, 18 años).

- Bienestar: el disfrutar la ejecución genera un mejor estado de ánimo y humor, produciendo bienestar en el ejecutante. Ejemplo: "una sensación de bienestar con uno mismo" (Masculino, 20 años), "me mantiene de buen humor" (Masculino, 28 años).

- Fluidez: el disfrutar de la ejecución produce un estado de olvidarse de uno mismo, de dejarse llevar. Ejemplo: "me permite olvidarme de mí misma" (Femenino, 24 años), "me satisface a un punto tal que me olvido dónde estoy y quién soy” (Masculino, 32 años).

Consideramos que las experiencias anteriormente mencionadas caracterizan el efecto que la experiencia de disfrute produce en el área cognitiva.

\section{Emocional}

- Libertad: el efecto de disfrutar la ejecución produce un sentimiento liberador. Ejemplo: "libertad, tocar sin que nadie me ponga trabas" (Masculino, 19 años), "siento que puedo liberarme y estar en contacto con mis sensaciones y emociones” (Masculino, 38 años).

- Relajación: el disfrutar de la ejecución genera un sentimiento de distención corporal. Ejemplo: "me relaja” (Masculino, 34 años), "libero tensiones” (Masculino, 59 años).

- Felicidad: el efecto del disfrute provoca un sentimiento de alegría y felicidad. Ejemplo: "genera felicidad" (Masculino, 20 años), "da alegría al alma" (Masculino, 30 años).

- Sensación corporal: el disfrutar de la ejecución genera diferentes sensaciones corporales. Ejemplo: "es una necesidad interna, me lo pide el cuerpo, está latente" (Masculino, 29 años), "energía y fuerza de voluntad" (Masculino, 20 años).

- Plenitud: el efecto de disfrutar la ejecución provoca una sensación de plenitud. Ejemplo: "es lo que me hace sentir más pleno, donde soy plenamente yo" (Masculino, 20 años), "me hace sentir absolutamente plena" (Femenino, 29 años).

- Producción propia: el efecto del disfrute se encuentra ligado al hecho de que se genera algo nuevo y de producción propia en la ejecución. Ejem- 
plo: "permite ver el potencial creativo que hay en uno" (Femenino, 24 años), "me permite poner en juego mi creatividad" (Femenino, 31 años).

- Expresión: el disfrutar de la ejecución permite encontrar en la música una forma de expresión diferente. Ejemplo: "poder expresar lo que uno tiene dentro" (Masculino, 21 años), "expresar cosas que de otra manera no podría" (Masculino, 27 años).

- Pasión: el efecto de disfrutar la ejecución es de sentimientos de amor por la música y por la ejecución en sí. Ejemplo: "el amor" (Masculino, 32 años), "pasión" (Masculino, 35 años).

- Placer: el disfrutar de la ejecución genera una sensación de placer. Ejemplo: "un efecto placentero, como el de jugar" (Masculino, 30 años), "un placer que es indescriptible" (Masculino, 29 años).

- Conexión con mundo interno: el disfrutar la ejecución genera la sensación de conectarse con uno mismo. Ejemplo: "me conecta más conmigo mismo" (Masculino, 20 años), "me permite encontrarme conmigo mismo" (Masculino, 27 años).

- Conexión con instrumento/música: el disfrutar de la ejecución produce una sensación de conexión con el instrumento y la música. Ejemplo: "encuentro gratificante pasar tiempo a solas con el instrumento, encontrando nuevos sonidos" (Masculino, 25 años), "todo lo que hago lo hago con ritmo" (Masculino, 21 años).

Consideramos que las experiencias anteriormente mencionadas caracterizan el efecto que la experiencia de disfrute produce en el área emocional.

\section{Motivacional}

- Motivación: el efecto del disfrute al ejecutar genera mayores ganas de seguir tocando y practicando. Ejemplo: "motiva a practicar para que siga creciendo esa emoción" (Masculino, 16 años), "me motiva a plantearme objetivos a corto y largo plazo" (Masculino, 28 años).

- Aprendizaje: el disfrute de la ejecución desarrolla la idea del proceso de aprendizaje, superación. Ejemplo: "satisfacción por el aprendizaje" (Masculino, 34 años), "siempre hacer y aprender algo nuevo" (Femenino, 26 años).

- Terapéutico: el efecto de disfrute es terapéutico, genera un estado físico y mental saludable. Ejemplo: "me mantiene sano física y emocionalmente" 
(Masculino, 58 años), “tiene un fuerte efecto terapéutico” (Masculino, 39 años).

- Profesional: el efecto del disfrute es de brindar una salida laboral, sostén económico a través de la actividad. Ejemplo: "puedo vivir de lo que más me gusta" (Masculino, 18 años), "me permite desarrollar lo que elegí para vivir, y a la vez utilizarlo como fuente laboral y de supervivencia" (Masculino, 36 años).

- Realización personal: el efecto del disfrute de la ejecución es de brindar identidad, define la personalidad, la idea de realizar lo que uno desea. Ejemplo: "donde soy plenamente yo" (Masculino, 20 años), "me siento realizada" (Femenino, 47 años).

- Sentido de vida: el disfrute en la ejecución le brinda sentido a la vida. Ejemplo: "es a lo que vine a este mundo, mi vida cobra significado" (Femenino, 29 años), "es el sentido y las ganas de seguir adelante, siempre" (Masculino, 24 años).

Consideramos que las experiencias anteriormente mencionadas caracterizan el efecto que la experiencia de disfrute produce en los aspectos motivacionales.

\section{Contextual}

- Conexión con otros: el efecto de disfrutar la ejecución es de generarse una conexión con el grupo o el público. Ejemplo: "conectar con otras personas desde un lugar de disfrute" (Femenino, 24 años), "compartir y brindarle la experiencia a los demás" (Femenino, 35 años).

Consideramos que las experiencias anteriormente mencionadas caracterizan el efecto que la experiencia de disfrute produce en los aspectos contextuales

\section{Definición de categorías} ban.

En cada categoría se observaron los componentes empíricos que la expresa-

A partir del análisis de contenido de las frases expresadas por los músicos definimos el disfrute como experiencia subjetiva positiva con diferentes componentes expresivos en las áreas cognitiva, emocional, motivacional y contextual, que constituyen las siguientes categorías de análisis. 


\section{Cognitiva}

Productos del procesamiento cognitivo de la experiencia de disfrute. Involucra la memoria, percepción del tiempo, atención, concentración, pensamientos y creencias acerca de cómo funciona la mente (metacogniciones). Objetos del pensamiento en relación a sí mismo, a la tarea, al rendimiento.

\section{Emocional}

Registro subjetivo de percepciones en relación a estados afectivos durante la experiencia de disfrute. Percepción, experiencia interna e inmediata de las sensaciones asociadas a la experiencia de disfrute. La referencia a las emociones puede darse desde el plano metafórico o imaginario (sentirse libre, estado de perfección) o en otro más anclado en las percepciones físicas de la experiencia (sensación de calma, relajación).

\section{Motivacional}

Involucra una tendencia a la acción, la inversión de tiempo real en la práctica de la ejecución musical. Implica la dirección e intensidad de nuestros propios esfuerzos y la causa de la performance/ejecución. Es una motivación principalmente intrínseca en una actividad significativa para el sujeto. Aspectos y razones que llevan a la ejecución del instrumento.

\section{Contextual}

Condiciones contextuales físicas, puesta en escena, escenario de la experiencia. ¿Dónde? ¿Cómo? ¿Con quién? Integra la interacción social de la performance. Implica el reconocimiento de la naturaleza cambiante y dinámica del contexto.

\section{Resultados}

\section{Categorías del disfrute en la ejecución musical y sus componentes expresivos}

Se observa que el efecto del disfrute en la ejecución del instrumento que más fue evocado es el de Felicidad (20,77\%), luego el de Relajación (12,07\%) y el de Placer $(10,62 \%)$. La mayor cantidad de referencias están ligadas a cuestiones emocionales. 
Luego se observa que dentro de las otras categorías, a nivel cognitivo, el efecto más mencionado es el de Tranquilidad (10,14\%), luego Bienestar (8,69\%), Satisfacción $(8,69 \%)$ y Despreocupación $(8,69 \%)$.

A nivel motivacional, el efecto más mencionado está ligado a la Realización personal (8,69\%), Aprendizaje (7,72\%) y Sentido de vida $(7,24 \%)$.

Encontramos que la conexión con otros es un componente de la categoría contextual.

Presentamos a continuación la relación de las categorías y sus componentes expresivos con otras variables.

\section{Relación de los componentes expresivos y la edad de los ejecutantes}

- Relación de los componentes expresivos de la categoría cognitiva y la edad de los ejecutantes: se observa que el componente concentración es mencionado sólo por ejecutantes de 16 a 35 años. El componente bienestar es el único que fue referido en todas las edades.

- Relación de los componentes expresivos de la categoría emocional y la edad de los ejecutantes: los componentes conexión con instrumento/música y plenitud fueron mencionados sólo por ejecutantes de 16 a 35 años. Felicidad es el único componente mencionado en todas las edades.

- Relación de los componentes expresivos de la categoría motivacional y la edad de los ejecutantes: se observa que el componente motivación sólo es mencionado por ejecutantes de 16 a 35 años. Sentido de vida sólo es referido por personas de 26 a 35 años.

- Relación de los componentes expresivos de la categoría contextual y la edad de los ejecutantes: la conexión con otros como efecto sólo es mencionada por ejecutantes entre los 16 y los 45 años. Los ejecutantes entre 46 y 65 años no refirieron efectos contextuales.

\section{Relación de los componentes expresivos y el sexo de los ejecutantes}

- Relación de los componentes expresivos de la categoría cognitiva y el sexo de los ejecutantes: se observa que hay marcadas diferencias en la cita de los componentes salvo en concentración. El componente satisfacción presenta mayores expresiones en los sujetos masculinos.

- Relación de los componentes expresivos de la categoría emocional y el sexo de los ejecutantes: son marcadas las diferencias por sexo en felicidad, 
placer y relajación. Los sujetos masculinos hacen referencia mayoritariamente.

- Relación de los componentes expresivos de la categoría motivacional y el sexo de los ejecutantes: sentido de vida, realización personal y aprendizaje son componentes básicamente mencionados por los sujetos masculinos.

- Relación de los componentes expresivos de la categoría contextual y el sexo de los ejecutantes: es muy pequeña la mención de efectos en la categoría contextual.

\section{Relación de los componentes expresivos y el tipo de instrumento que tocan los ejecutantes}

- Relación de los componentes expresivos de la categoría cognitiva y el tipo de instrumento que tocan los ejecutantes: los ejecutantes de instrumentos de cuerda son los que presentan mayor número de efectos en todos los componentes.

- Relación de los componentes expresivos de la categoría emocional y el tipo de instrumento que tocan los ejecutantes: los ejecutantes de instrumentos de cuerda presentan la mayor cantidad de efectos en los componentes de esta categoría, destacándose felicidad, relajación y expresión.

- Relación de los componentes expresivos de la categoría motivacional y el tipo de instrumento que tocan los ejecutantes: sentido de vida fue mencionado básicamente por ejecutantes de cuerdas.

- Relación de los componentes expresivos de la categoría contextual y el tipo de instrumento que tocan los ejecutantes: conexión con otros sólo fue mencionada por ejecutantes de viento, cuerdas y percusión.

Con relación a la edad, de 16 a 25 años y de 26 a 35, expresaron mayores efectos cognitivos, emocionales, motivacionales y contextuales.

Con relación al sexo, los ejecutantes masculinos expresaron mayores efectos en las cuatro categorías.

Con relación a los instrumentos que ejecutan, los instrumentistas de cuerdas son los que más referencia hacen a los componentes en las categorías cognitiva, emocional y motivacional.

Los efectos contextuales fueron escasamente nombrados por instrumentistas de percusión, viento y cuerdas. 
Los instrumentistas de cuerdas se destacan, con relación a demás, por referenciar a más componentes como efecto del disfrute en las distintas categorías.

\section{Conclusiones}

Generamos un modelo conceptual y establecimos relaciones entre las categorías surgidas de los datos (figura 2). Estas relaciones están definidas respecto del disfrute, desde la percepción de los ejecutantes de instrumentos musicales de este estudio. Pudimos identificar las experiencias psicológicas como componentes expresivos en cada categoría (ver resultados).

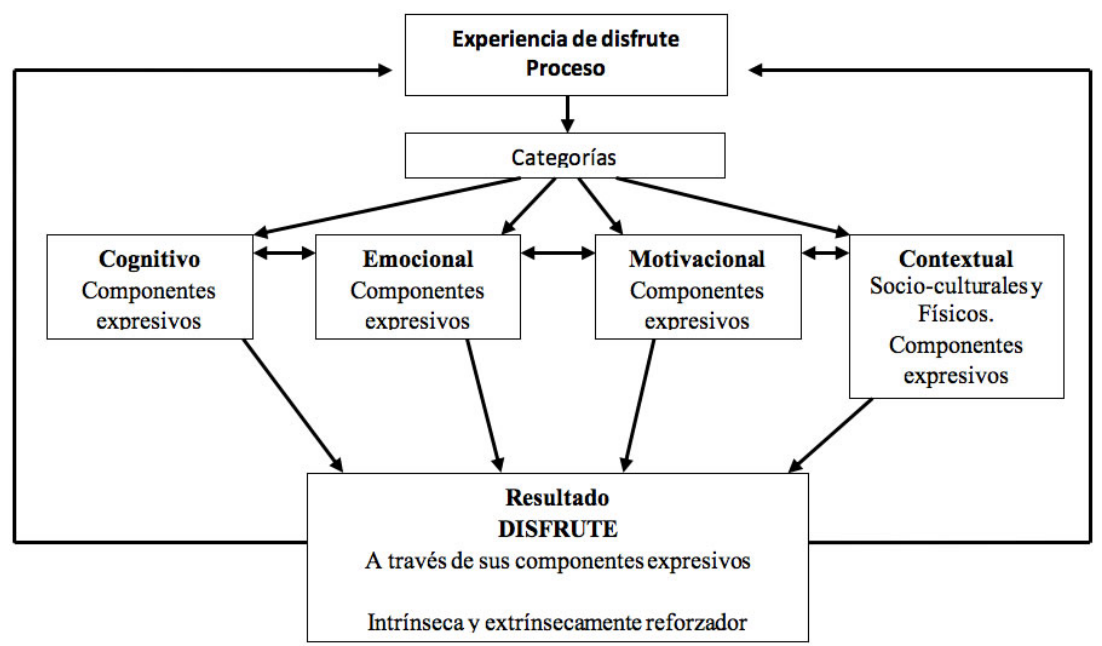

Figura 2. Esquema conceptual propuesto.

Hemos observado, tal como dijimos anteriormente y en esta muestra de ejecutantes, el disfrute aparece como una disposición positiva general con expresiones afectivas, cognitivas y motivacionales tal como ha sido señalado por Nabi y Krcmar (2004).

Los efectos del mismo se expresan través de componentes empíricos que refieren a una experiencia psicológica particular.

Los dos rangos de edad en los que encontramos mayores elementos expresivos de los efectos del disfrute a nivel cognitivo, emocional y motivacional están 
constituidos por personas jóvenes de entre 16 a 35 años. ¿Esto quiere decir que los efectos del disfrute y la experiencia misma de disfrute tienen más componentes expresivos en esta edad? ¿O que los más jóvenes reportan con mayor detalle la experiencia de disfrute vivida? Asimismo, son más personas en la muestra los de esta edad, por lo que puede llevarnos a asunciones erradas atribuirle cualidades distintivas en esta edad a la experiencia de disfrute, cuando lo que estamos haciendo es una categorización y un análisis de las veces que esa respuesta aparece. No nos habla de las cualidades inherentes de la experiencia, sino de las formas de referirse a ella por parte del grupo mayoritario de esta muestra.

Sin embargo, resulta de interés conocer en detalle cómo las personas que ejecutan instrumentos musicales expresan los efectos que tiene en su vida el disfrute en la ejecución musical. A nivel cualitativo la cuestión más destacable es que el efecto del disfrute en la ejecución de un instrumento musical se encuentra significado como felicidad y relajación en el área emocional, mientras que tranquilidad, bienestar, satisfacción y despreocupación caracterizan el efecto del disfrute en el área cognitiva. A nivel motivacional se ha consignado que el efecto del disfrute en la ejecución musical es la realización personal principalmente. Estas cuestiones han sido rescatadas de los dichos de los y las participantes de la presente investigación y expresan estados complejos. Cada una de las maneras de consignar los efectos del disfrute resultan complejas en sí mismas y cada una de ellas se presenta como estados psicológicos complejos y en ningún caso unidimensionales.

¿Podríamos pensar que los efectos del disfrute en la ejecución de instrumentos musicales son principalmente emocionales y cognitivos?

Si hemos definido de manera general al disfrute como una disposición positiva general con expresiones afectivas, cognitivas y motivacionales, podemos aquí afinar nuestras afirmaciones concluyendo que a nivel empírico en esta muestra de sujetos los efectos se expresan con los descriptores (componentes expresivos) que hemos mencionado anteriormente.

Otro aporte de la presente investigación es tratar de enriquecer cada una de las categorías propuestas como parte del disfrute en la ejecución de instrumento musical más allá del agrado o de la proposición de polos opuestos entre emociones de afecto positivo, en contraposición a emociones negativas. El reporte de la experiencia a través de los diversos componentes expresivos, hallados desde la visión de las propias personas que la vivencian, nos permite enriquecer la gama de expresiones que pueden describirlas y entender el significado del disfrute en la ejecución de instrumentos musicales (DeNora, 2000), además de la escucha.

Así nos corremos de posiciones dicotómicas (me gusta/no me gusta) para 
comprender más acabadamente al disfrute como un fenómeno psicológico complejo, cuyo efecto está caracterizado por emociones y afectividad compleja (como la felicidad) con correlatos sensitivo/corporales (como la relajación y el placer), así como por aspectos cognitivos relacionados a la preocupación/ansiedad (tranquilidad y despreocupación), a percepciones más generalizadas (como el bienestar) y a juicios (como la satisfacción).

Este estudio exploratorio es el comienzo de un largo camino en relación a los estados psicológicos que transcurren durante la performance de los instrumentistas. Sabemos que hay mucho por investigar y ello constituye un gran desafío para continuar en esta línea. Para Finnegan (1989) en la investigación etnometodológica, la música se concibe más como un modo de interacción (para nosotros formando parte de la categoría contextual) que como un efecto de la percepción auditiva. Este modo de interacción a través de la ejecución en muchos casos produce disfrute.

\section{Referencias}

Buscatto, M. (2016). Women in artistic professions. An emblematic paradigm for gender studies. Social Cohesion and Development, 2(1), 69-77. doi: 10.12681/scad.9040

Csikszentmihalyi, M., y Csikszentmihalyi, I. (1975). Beyond boredom and anxiety. San Francisco: Jossey-Bass.

Csikszentmihalyi, M. (1993). The Evolving evolving self: A psychology for the third millennium. Barcelona: Kairós.

Csikszentmihalyi, M., y Csikszentmihalyi, I. (Eds.). (1988). Optimal Experience: Psychological Studies of Flow in Consciousness. Nueva York: Cambridge University Press.

Delle Fave, A., Massimini, F., y Bassi, M. (2011). Psychological selection and optimal experience across cultures. Social empowerment through personal growth. Nueva York: Springer.

DeNora, T. (2000). Music in everyday life. Cambridge: Cambridge University Press

Finnegan, R. (1989). The bidden musicians: Music-making in an english town. Cambridge: Cambridge University Press.

Hernández Sampieri, R., Fernández-Collado, C., y Baptista Lucio, P. (2014). Metodología de la investigación. 6ta edición. México: McGraw Hill.

Juslin, P. (2016). Emotional reactions to music. En S. Hallam, I. Cross, y M. Thaut, The Oxford Handbook of Music Psychology (pp. 197-213). 2da edición. Oxford: Oxford University Press. 
Kohut, H., y Levarie, S. (1950). On the enjoyment of listening to music. The Psychoanalytic Quarterly, 19(1), 64-87. doi: 10.1080/21674086.1950.11925787

Lamont, A. (2011). University students strong experience of music: Pleasure, engagement and meaning. Musicae Scientiae, 15(2), 229-249. doi: 10.1177/102986491101500206

Leibovich de Figueroa, N., y Schmidt, V. (2010). Ecoevaluación psicológica en el contexto familiar. Aportes conceptuales. En N. Leibovich de Figueroa y V. Schmidt (Eds.), Ecoevaluación psicológica del contexto familiar. Aspectos teóricos y empiricos. Adaptación argentina de escala Faces III (pp. 13-25). Buenos Aires: Guadalupe.

Moon, J., y Kim, Y. (2001). Extending the TAM for a World Wide Web context. Information \& management, 38(4), 217-230. doi: 10.1016/S0378-7206(00)00061-6

Nabi, R. L., y Krcmar, M. (2004). Conceptualizing media enjoyment as attitude: Implications for mass media effects research. Communication Theory, 14(4), 288-310. doi: 10.1111/j.1468-2885.2004.tb00316.x

O’Neill, S. A., y Boultona, M. J. (1996). Boys' and girls' preferences for musical instruments: A function of gender? Psychology of Music, 24(2), 171-183. doi: $10.1177 / 0305735696242009$

Schneider, H. (1921). The enjoyment of music. The Musical Quarterly, 7(2), 218-225. doi: $10.1093 / \mathrm{mq} /$ VII.2.218

Schubert, E. (1996). Enjoyment of negative emotions in music: An associative network explanation. Psychology of Music, 24(1), 18-28. doi: 10.1177/0305735696241003

Sloboda, J. A., O’Neill, S. A., y Vivaldi, A. (2001). Functions of music in everyday life: an exploratory study using the Experience Sampling Method. Musicae Scientiae, 5(1), 9-32. doi: $10.1177 / 102986490100500102$

Williams, D. M., Papandonatos, G. D., Napolitano, M. A., Lewis, B. A., Whiteley, J. A., y Marcus, B. H. (2006). Perceived enjoyment moderates the efficacy of an individually tailored physical activity intervention. Journal of Sport and Exercise Psychology, 28(3), 300-309. doi: 10.1123/jsep.28.3.300 


\title{
Acerca de las autoras
}

\section{Nora Leibovich}

\author{
nleibovich@fibertel.com.ar
}

Miembro de la Carrera del Investigador Científico (CONICET) en la categoría Investigador Principal. Investigadora categoría "I", Secretaría de Ciencia y Técnica, Universidad de Buenos Aires. Profesora Emérita de la Universidad de Buenos Aires. Directora de la Carrera de Especialización en Estrategias de Evaluación en Psicología en la Universidad Abierta Interamericana (UAI). Ex Directora Carrera de Especialización en Evaluación Psicológica. Premio a la excelencia académica 2015 por sus 50 años de Docencia ininterrumpida en la Universidad de Buenos Aires. Ha publicado numerosos libros y papers sobre evaluación psicológica, el estudio del estrés, de la inestabilidad laboral y otros grandes aportes a la psicología. 